\title{
A Lagrangian Advection Scheme for Solving Cloud Droplet Diffusion Growth
}

\author{
Lei Wei ${ }^{1,2, *}$, Jiming Sun ${ }^{3,4,5, *}$, Hengchi Lei ${ }^{3}$, Li Dong ${ }^{3}$ and Wenhao $\mathrm{Hu}^{3,4}$ \\ 1 Beijing Weather Modification Office, Beijing 100089, China \\ 2 Beijing Key Laboratory of Cloud, Precipitation and Atmospheric Water Resources, Beijing 100089, China \\ 3 Institute of Atmospheric Physics, Chinese Academy of Sciences, Beijing 100029, China; \\ leihc@mail.iap.ac.cn (H.L.); dongli@lasg.iap.ac.cn (L.D.); huwenhao@mail.iap.ac.cn (W.H.) \\ 4 College of Earth and Planetary Sciences, University of Chinese Academy of Sciences, Beijing 100049, China \\ 5 Nanjing University of Information Science and Technology, Nanjing 210044, China \\ * $\quad$ Correspondence: weilei@mail.iap.ac.cn (L.W.); jimings@mail.iap.ac.cn (J.S.); Tel.: +86-10-68400864 (L.W.)
}

Received: 21 March 2020; Accepted: 12 June 2020; Published: 15 June 2020

check for

Abstract: Cloud drop diffusion growth is a fundamental microphysical process in warm clouds. In the present work, a new Lagrangian advection scheme (LAS) is proposed for solving this process. The LAS discretizes cloud drop size distribution (CDSD) with movable bins. Two types of prognostic variable, namely, bin radius and bin width, are included in the LAS. Bin radius is tracked by the well-known cloud drop diffusion growth equation, while bin width is solved by a derived equation. CDSD is then calculated with the information of bin radius, bin width, and prescribed droplet number concentration. The reliability of the new scheme is validated by the reference analytical solutions in a parcel cloud model. Artificial broadening of CDSD, understood as a by-product of numerical diffusion in advection algorithm, is strictly prohibited by the new scheme. The authors further coupled the LAS into a one-and-half dimensional (1.5D) Eulerian cloud model to evaluate its performance. An individual deep cumulus cloud studied in the Cooperative Convective Precipitation Experiment (CCOPE) campaign was simulated with the LAS-coupled 1.5D model and the original 1.5D model. Simulation results of CDSD and microphysical properties were compared with observational data. Improvements, namely, narrower CDSD and accurate reproduction of particle mean diameter, were achieved with the LAS-coupled 1.5D model.

Keywords: Lagrangian advection scheme; cloud drop diffusion growth; cloud models

\section{Introduction}

Warm clouds have been an ongoing research interest in the field of cloud physics, weather forecasting, and climate change for decades. The classic warm cloud theory indicates that cloud droplets in a cloudy air parcel, which originally form upon hygroscopic cloud condensation nuclei $(\mathrm{CCN})$ through heterogeneous nucleation, continue to grow by water vapor diffusion until their radii reach approximately $20 \mu \mathrm{m}$ to serve as warm rain embryos and initiate the collision-coalescence process. Of all the microphysical processes covered by warm clouds, cloud droplet diffusion growth is fundamental. Cloud droplet diffusion growth dominates the evolution of cloud droplet size distribution (CDSD) in the early developing stage of cumulus clouds, which influences the rate of precipitation formation and cloud lifetime [1]. Additionally, buoyancy produced by latent heat release due to cloud droplet diffusion growth is a main driving force for cloudy air parcel acceleration [2], as confirmed by multiple numerical case studies [3,4]. Cloud models based on various approaches have been developed to reproduce the cloud droplet diffusion growth process and further investigate its characteristics. Two main approaches describing cloud microphysical properties and processes have been developed: bulk parameterization models and spectral bin models [5-7]. 
A priori information about the shape of the cloud droplet size distribution is required by all bulk parameterization schemes, and a gamma distribution with four independent parameters is adopted by most schemes (the gamma distribution function is $\mathrm{f}(\mathrm{m})=\mathrm{N}_{0} \mathrm{~m}^{\nu} \exp \left(-\lambda \mathrm{m}^{\mu}\right)$, where $N_{0}$ is the intercept, $v$ is the shape parameter, $\lambda$ is the slope parameter, and $\mu$ is the dispersion parameter). An outstanding benefit of bulk schemes is the low computational cost. However, there are some unresovled issues with these schemes. The accuracy of using a gamma function to represent the droplet size distributions of different types of hydrometeors and their evolution have been argued to be quite limited, even though multiple measures have been applied to improve the representation [8-11]. This limitation, to some degree, can be revealed in a straightforward manner. In the simulation of cloud droplet spectra under a constant supersaturation with a double-moment bulk scheme, the CDSDs tend to maintain a rather broad shape by diffusion growth over time; however, narrow CDSDs should be guaranteed as dictated by the diffusion growth law.

In contrast to bulk schemes, Eulerian spectral bin cloud models discretize the mass of each type of hydrometeor into a number of fixed bins ranging from tens to thousands, and the shape of a droplet spectrum is determined by the droplet number concentration of each individual bin instead of the parameters of the distribution function. The diffusion growth rate of cloud droplets for each bin can be explicitly obtained by solving the well-known diffusional growth equations [12]. Although it is affected by multiple factors, the accuracy of a spectral bin scheme is closely related to the resolution of the bins. Multifold considerations, including the characteristics of the research object, will be weighed by a cloud physicist when choosing the number of bins to use. For example, a 2000-bin parcel cloud model was used to study the sensitivity of precipitation to atmospheric aerosols in cumulus clouds [13]. A concurrent and unresolved issue with spectral bin models is the spurious DSD broadening due to the remapping procedure of cloud droplets between adjacent fixed bins after calculating the diffusion growth at each timestep [14]. This issue can be reduced at the cost of expensive computational resources by increasing the resolution of the bins.

The past decade witnessed rapid development in Lagrangian cloud models (LCMs) featuring the dynamic and microphysical behaviors of droplets that can be directly tracked. The LCM is also claimed to be the most natural framework to study cloud droplet and raindrop development [15]. One major group of LCMs employs the superdroplet approach [16-18], where a superdroplet is defined as a group of droplets sharing the same attributes. A Lagrangian drop model based on the superdroplet approach has also been introduced [15]. These methods have been coupled to large eddy simulation (LES) models and applied to investigate warm cloud processes. Lagrangian approaches are reported to be superior in terms of computational costs over a Eulerian scheme [19]. The other group of LCMs employs a direct numerical simulation (DNS) approach (e.g., Onishi et al., 2015; Saito et al., 2018; Chen et al., 2018) [20-22]. DNS methods can simulate the motion and growth of each droplet directly within a limited box area and are appealing tools to study the effects of turbulence on cloud droplet collision efficiency. Recently, some breakthroughs have been made, bridging all the key parts of warm rain processes in DNS models [23].

Notwithstanding all the remarkable models mentioned above, faithfully reproducing microphysical processes remains challenging. New schemes, approaches, and models urgently need to be developed. Following the framework of movable mass grids in a parcel cloud model [24] and the algorithms of a Lagrangian advection scheme (LAS) for air parcels [25], we have developed a new LAS for solving cloud drop diffusion growth. The Lagrangian bins are movable as the mass of cloud droplets changes. In addition, the width of each bin will evolve as the bin moves, which was achieved by solving a prognostic equation derived from the advection-type continuity equation for the CDSD projected in Lagrangian view. A parcel cloud model was used to verify the newly developed Lagrangian advection scheme. In order to explore the potential applications of the new scheme in more complicated cloud models, we further coupled it into a one-and-half dimensional Eulerian cloud-aerosol interaction bin model (1.5D model) in a hybrid manner. Numerical convection tests with the 1.5D model were conducted to evaluate the new scheme. 


\section{Methods}

The framework of the new Lagrangian advection scheme is described in this section in detail. In cloud physics, the advection equation describing the evolution of the CDSD through diffusion growth was given by Rogers and Yau (1989) [26]. If we discretize the CDSD into bins, the equation can be written as follows:

$$
\frac{\partial \mathrm{f}_{\mathrm{i}}}{\partial \mathrm{t}}+\frac{\partial}{\partial \mathrm{r}_{\mathrm{i}}}\left(\mathrm{f}_{\mathrm{i}} \frac{\mathrm{dr}}{\mathrm{dt}}\right)=0
$$

where $t$ is time, $f_{i}$ denotes the number density of particles with radius $r_{i}, f_{i} d r_{i}$ is the number of particles per unit volume air between radius $r_{i}$ and $r_{i}+d r_{i}$, and the subscript $i$ represents a certain bin.

In Eulerian spectral bin cloud models, finite-difference algorithms are adopted to solve Equation (1) on fixed meshes (bins). The mass flux through the cell (bin) edges will be computed, and depending on the algorithms chosen and the order of accuracy selected, numerical solutions will suffer from a degree of dispersion. Numerical dispersion may cause artificial broadening of the cloud droplet spectrum and alter the lifetime of a cumulus cloud by triggering the collision process in advance [27].

In the Lagrangian framework, the previous partial differential equation is transformed into the following differential equation:

$$
\frac{d f_{i}}{d t}+f_{i} \frac{\partial}{\partial r_{i}}\left(\frac{d r_{i}}{d t}\right)=0
$$

Consistent with the methods adopted in Lagrangian parcel models, the CDSD is discretized into movable bins in the LAS. Each bin represents a number of cloud droplets with an identical radius and will be tracked all the time as the cloud droplets experience diffusion growth or evaporation. The equation for diffusion growth is directly solved on movable bins. Therefore, remapping is no longer needed, and no artificial broadening of the CDSD will be introduced.

In addition to advancing the radius of each movable bins, the LAS explicitly predicts the time evolution of bin width by solving a prognostic equation. The droplet number concentration as a conserved quantity is used to derive the prognostic equation for bin width. The droplet number concentration can be expressed as follows:

$$
\mathrm{f}_{\mathrm{i}}=\frac{\mathrm{n}_{\mathrm{i}}}{\Delta \mathrm{r}_{\mathrm{i}}}
$$

where $n_{i}$ is the number concentration in the ith bin, and $\Delta r_{i}$ is the width of the ith bin. Figure 1 gives a schematic illustration of the Lagrangian bins. Combining Equation (3) with Equation (2), the core equation set for the LAS is as follows:

$$
\begin{gathered}
\frac{\mathrm{d} \Delta \mathrm{r}_{\mathrm{i}}}{\mathrm{dt}}=\Delta \mathrm{r}_{\mathrm{i}} \frac{\partial}{\partial \mathrm{r}_{\mathrm{i}}}\left(\frac{\mathrm{dr}_{\mathrm{i}}}{\mathrm{dt}}\right) \\
\frac{\mathrm{dr} \mathrm{r}_{\mathrm{i}}}{\mathrm{dt}}=\mathrm{v}_{\mathrm{i}}
\end{gathered}
$$

where the droplet diffusion growth rate is $\mathrm{v}_{\mathrm{i}}$. Both the cloud droplet radius $\mathrm{r}_{\mathrm{i}}$ and the width of the bin $\Delta r_{i}$ will change over time under certain supersaturation, temperature, and pressure conditions. In the context of fluid dynamics or atmospheric dynamics, the counterpart of Equation (5) is usually called the trajectory equation for mobile air parcels. In the context of cloud physics, Equation (5) is the well-understood diffusion growth equation, whose value is inversely proportional to the droplet radius. Utilizing this rule, Equation (4) can be further transformed to eliminate the partial differential term on the right-hand side:

$$
\frac{\mathrm{d} \Delta \mathrm{r}_{\mathrm{i}}}{\mathrm{dt}}=-\frac{\Delta \mathrm{r}_{\mathrm{i}}}{\mathrm{r}_{\mathrm{i}}}\left(\frac{\mathrm{dr} \mathrm{r}_{\mathrm{i}}}{\mathrm{dt}}\right)
$$

and the numerical solution of Equation (6) can be obtained in a straightforward way. It should be noted that the prognostic variable in the advection equation governing the evolution of CDSD transforms 
from droplet number concentration $\mathrm{f}_{\mathrm{i}}$ to bin width $\Delta \mathrm{r}_{\mathrm{i}}$ in the new Lagrangian scheme. The CDSD at a certain moment can therefore be diagnosed with the bin width.

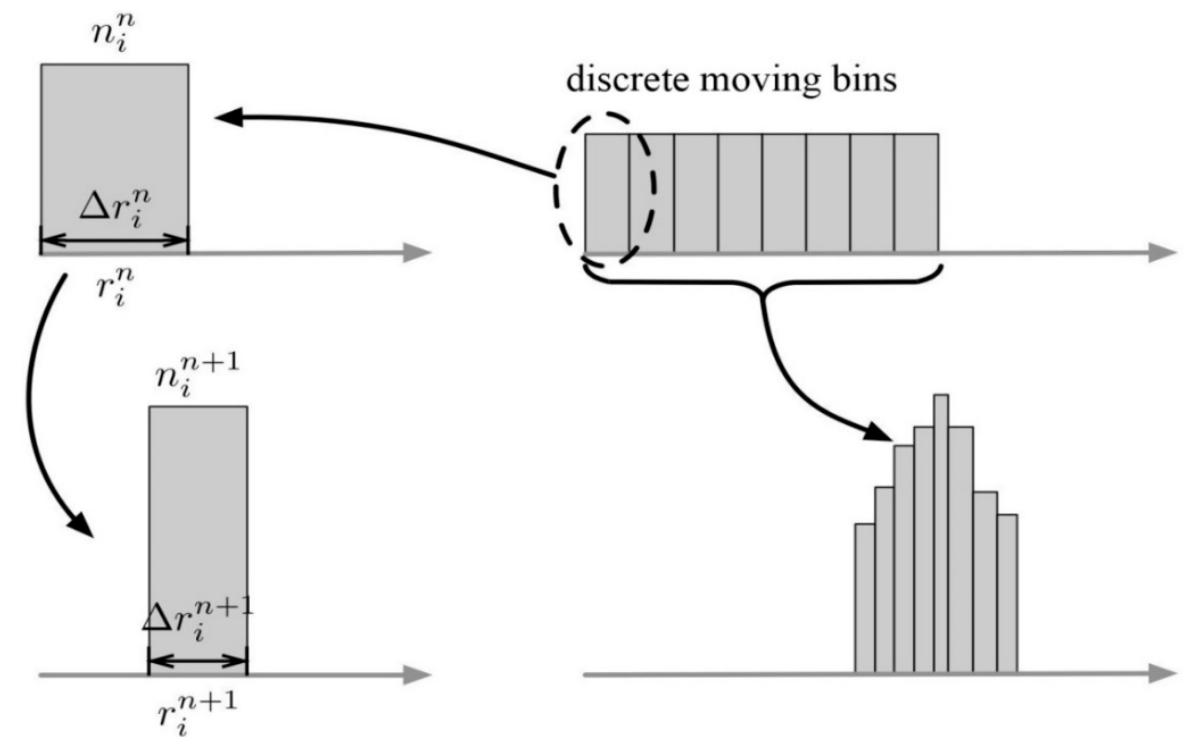

Figure 1. Diagram of the time evolution of the discrete moving bins $\left(n_{i}^{n}=n_{i}^{n+1}\right.$, where the superscript of $n_{i}$ is from the timestep $n$ to $\left.n+1\right)$.

\section{Experiments}

\subsection{Numerical Tests in a Parcel Cloud Model}

A parcel cloud model was used to assess the Lagrangian advection scheme. Numerical tests using parcel cloud model with the Lagrangian advection scheme and a Eulerian advection scheme (EAS) were performed. Simulation results from both schemes were compared and are discussed in the next section. Reference analytical solutions were also introduced to examine these results.

The parcel cloud model employed a gamma distribution function as the initial cloud drop spectrum. The thermodynamic and microphysical setups were as follows. The supersaturation was fixed at $0.1 \%$, the temperature was $285.15 \mathrm{~K}$, and the pressure of the ambient air was $800 \mathrm{hPa}$. The cloud droplet number concentration was $200 \mathrm{~cm}^{-3}$, and the mass of the first bin was $4.0 \times 10^{-15} \mathrm{~g}$. Two types of advection schemes were coupled into the parcel model: the Eulerian advection scheme and the Lagrangian advection scheme. Different algorithms were applied to obtain the numerical solutions. The Eulerian advection scheme employed was the second-order-accurate flux-corrected transport (FCT) variant of the multidimensional positive definite advection transport algorithm (MPDATA) scheme [28,29] for cloud drop advection in spectral space. The Lagrangian advection scheme was advanced using a fourth-order Runge-Kutta method.

The diffusion growth equation solved in the parcel cloud model was taken from Rogers and Yau (1989), ignoring the solution terms and curvature terms. The equation can be written as follows:

$$
\frac{\mathrm{dr}}{\mathrm{dt}}=\frac{\mathrm{S}-1}{\frac{\rho_{\mathrm{L}} R_{\mathrm{v}} \mathrm{T}}{\mathrm{D}_{\mathrm{v}} \mathrm{e}_{\mathrm{S}}(\mathrm{T})}+\frac{\mathrm{L} \rho_{\mathrm{L}}}{\mathrm{KT}}\left(\frac{\mathrm{L}}{\mathrm{R}_{\mathrm{v}} \mathrm{T}}-1\right)} \mathrm{r}^{-1}
$$

where $S=e / e_{s}(T)$ is the ambient saturation ratio, e is the water vapor pressure, $e_{s}(T)$ is the saturation vapor pressure, supersaturation is defined as $S-1, \rho_{\mathrm{L}}$ is the density of the water, $\mathrm{T}$ is the ambient temperature, $\mathrm{L}$ is the specific latent heat of evaporation, $D_{\mathrm{v}}$ is the molecular diffusion coefficient, $R_{\mathrm{v}}$ the universal gas constant, $\mathrm{r}$ is the radius of the cloud particles, and $\mathrm{K}$ is the coefficient of the thermal conductivity of air. 
The analytical solutions of cloud drop condensation growth, considered as the "true value", were employed to evaluate the two schemes. With solution terms and curvature terms ignored, the analytical solutions can be obtained by the following equations [26]:

$$
\begin{gathered}
r(t)=\sqrt{r_{0}^{2}+2 \frac{S-1}{F_{k}+F_{d}} t} \\
F_{k}=\frac{L \rho_{L}}{K T}\left(\frac{L}{R_{v} T}-1\right) \\
F_{d}=\frac{\rho_{L} R_{v} T}{D_{v} e_{s}(T)}
\end{gathered}
$$

where $F_{k}$ represents the thermodynamic term in the denominator that is associated with heat conduction, and $F_{d}$ is the term associated with vapor diffusion.

\subsection{Numerical Tests Based on the 1.5D Model}

\subsubsection{Model Description}

A one-and-half dimensional Eulerian cloud-aerosol interaction bin model [30] was applied to further investigate the performance of the LAS. The model included two circular concentric air columns, where the inner column described the updraft/cloud region and the outer column addressed the compensating downward motion region. The dynamic framework was essentially one-dimensional but allowed for an entrainment process by considering an external cylinder around the one-dimensional cloud region [31]. The model included both liquid-phase microphysics and ice-phase microphysics using 90 and 130 bins to describe the distributions of aerosols and hydrometeors, respectively. Although computationally costly, multidimensional particle size distributions (PSDs) were adopted by the model. Therefore, not only the interaction between the cloud droplets and aerosol particles but also the dry aerosol mass within each cloud drop could be explicitly treated. In this part, only the aerosol activation process and cloud drop condensation (evaporation) process were preserved, and all other microphysical processes were blocked. The dynamic timestep was set to $2 \mathrm{~s}$, and the timestep for microphysics was $0.2 \mathrm{~s}$. A detailed description of the $1.5 \mathrm{D}$ model can be found in the thesis of Sun (2008) [32].

\subsubsection{Condensation Growth in the Eulerian Framework}

The diffusion growth rates $(\mathrm{dm} / \mathrm{dt})_{\text {wat }}$ were given by Pruppacher and Klett (1997):

$$
\begin{gathered}
\left.\frac{\mathrm{dm}}{\mathrm{dt}}\right|_{\mathrm{wat}}=4 \pi \mathrm{r} \frac{\mathrm{s}_{\mathrm{V}, \mathrm{w}} \overline{\mathrm{f}}_{\mathrm{v}, \mathrm{L}^{*}}}{\frac{\rho_{\mathrm{w}} R T}{\mathrm{e}_{\mathrm{sat}, \mathrm{w}(\mathrm{T})} \mathrm{D}_{\mathrm{v}}^{\prime} \mathrm{M}_{\mathrm{W}}}+\frac{\mathrm{L}_{\mathrm{e}} \rho_{\mathrm{w}}}{\mathrm{k}_{\mathrm{aT}}^{\prime}}\left(\frac{\mathrm{L}_{\mathrm{e}} \mathrm{M}_{\mathrm{w}}}{\mathrm{RT}}-1\right)} \\
\mathrm{s}_{\mathrm{V}, \mathrm{w}}=\frac{\mathrm{e}}{\mathrm{e}_{\mathrm{sat}, \mathrm{w}}}-\exp (\mathrm{Y})
\end{gathered}
$$

where $\mathrm{m}$ is the mass of moist aerosol particles or cloud drops, $\mathrm{s}_{\mathrm{v}, \mathrm{w}}$ is the supersaturation, $\rho_{\mathrm{w}}$ is the density of water, $\mathrm{L}_{\mathrm{e}}$ is the specific latent heat of evaporation, $\mathrm{D}_{\mathrm{v}}^{\prime}$ is the modified diffusivity, $\mathrm{R}$ is the universal gas constant, $e$ is the actual water vapor pressure, $e_{s a t, w}$ is the saturation vapor pressure, $r$ is the radius of moist aerosol particles or cloud drops, $\mathrm{k}_{\mathrm{a}}^{\prime}$ is the modified thermal conductivity, $\mathrm{M}_{\mathrm{w}}$ is the molecular weight of water, and $\overline{\mathrm{f}}_{\mathrm{V}, \mathrm{L}}$ is the ventilation coefficient. Note that both the solution term and curvature term are neglected in Equation (12) $(\mathrm{Y}=0)$.

The Eulerian advection scheme adopted in the 1.5D model was the second-order-accurate FCT variant of MPDATA [28,29] for the advection of aerosol particles and cloud drops in spectral space.

The advance of the cloud drop spectrum distribution due to condensation growth in each timestep consisted of two steps. First, the condensation growth rate in each bin was computed using 
Equation (11), which served as the advection velocity in the following step. Second, the advection Equation (1) was solved using the MPDATA scheme to update the droplet number density in each bin.

\subsubsection{Condensation Growth in the Lagrangian Framework}

In the Eulerian framework, both microphysical processes and dynamical processes are all solved on fixed grids or bins. However, adjustments to the model framework were necessary when LAS was coupled into the 1.5D model. Specifically, the condensation growth was computed on the moving bins, whereas the dynamical processes were advanced on fixed meshes as the original model. A hybrid Eulerian-Lagrangian methodology was proposed to bridge the dynamic and microphysical processes. The basic idea was to compute the cloud drop condensation growth with high-resolution moving bins and then interpolate (remap) the cloud drop concentration in each moving bin to the relatively coarse fixed bins. Next, the dynamical processes were advanced on the fixed grids or bins. Finally, we quantified the variation in the cloud drop number concentration in the fixed bins and interpolated these results back to the moving bins for the next timestep. The number concentration of the cloud drop was strictly conserved in this process.

The calculation steps for cloud droplet condensation growth were as follows. Firstly, the time derivative of the droplet radius $(\mathrm{dr} / \mathrm{dt})$ on a moving bin was computed by Equation (11) with an additional assumption that all particles were spherically introduced. Then, the advection Equation (6) was solved to update the width of the moving bins $\Delta \mathrm{r}_{\mathrm{i}}$. Finally, the number density on each bin was advanced by Equation (3).

\subsubsection{Simulations of a Deep Cumulus Cloud in Cooperative Convective Precipitation Experiment (CCOPE) Campaign}

An individual continental-type deep cumulus cloud was simulated with the LAS-coupled 1.5D model and the EAS-coupled 1.5D model. The convection case we chose for the numerical tests was from the CCOPE field campaign. Specifically, the deep convection occurred at around 1610 Mountain Daylight Time (MDT), 19 July 1981, adjacent to Mile City, Montana [33]. Microphysical processes included in the model were CCN nucleation, condensation or evaporation, and collision-coalescence, with ice-phase processes omitted. The simulation results were compared with observational data. The aim of this numerical experiment was to evaluate the Lagrangian advection scheme against the Eulerian advection scheme for a deep cumulus case study.

The 1440 MDT sounding on 19 July 1981 at Mile City, Montana, was used as the sounding profile input data. A sinusoidal distribution of vertical velocity was prescribed below cloud base to initiate the convection. The observational data we used were collected by a National Oceanic and Atmospheric Administration (NOAA) sailplane (Tail Number N9929J), which penetrated the cumulus from cloud base $(3.9 \mathrm{~km}$ ) at about 1619 MDT and made spiral ascent to $7.2 \mathrm{~km}$ (about $1630 \mathrm{MDT}$ ) in the updraft region [33]. Cloud drops were sampled by a forward scattering spectrometer probe (FSSP) instrumented on the sailplane. The aircraft data we used in this study were extracted from the figures of Dye et al. (1986) [33].

\section{Results and Discussion}

\subsection{Parcel Cloud Model}

Figure 2 shows the time evolution of the cloud drop spectra from the analytical and numerical solutions. Cloud drops with radii smaller than $8 \mu \mathrm{m}$ made up the main body of the initial spectra, which resulted in rapid growth in the first $5 \mathrm{~min}$ of the simulations. The spectra moved rightward and became narrower, as shown in Figure 2a. By referring to the raw output data from the models, the peaks of the spectra moved from $0.8 \mu \mathrm{m}$ at the initial time to about $8.0 \mu \mathrm{m}$ at $5 \mathrm{~min}$. The peaks of the spectra from EAS (both 130 and 2000 bins) were located at the right-hand side of those from the LAS and the analytical solutions, indicating the cloud drops grew slightly faster with the EAS. It should be 
noted that the spectra from the LAS were much narrower than those from the EAS, which could also be observed in the following times. The shape and position of the cloud drop spectra with the LAS was in good accordance with the reference analytical solutions, indicating the results produced by the LAS were reliable. A major advantage of the LAS over the EAS was that the former scheme did not suffer the cloud drop spectrum spurious broadening issue. Simulations results from the EAS with 130 and 2000 bins also showed that the spurious broadening of the cloud drop spectra could be alleviated but not eliminated by increasing the bin resolution.
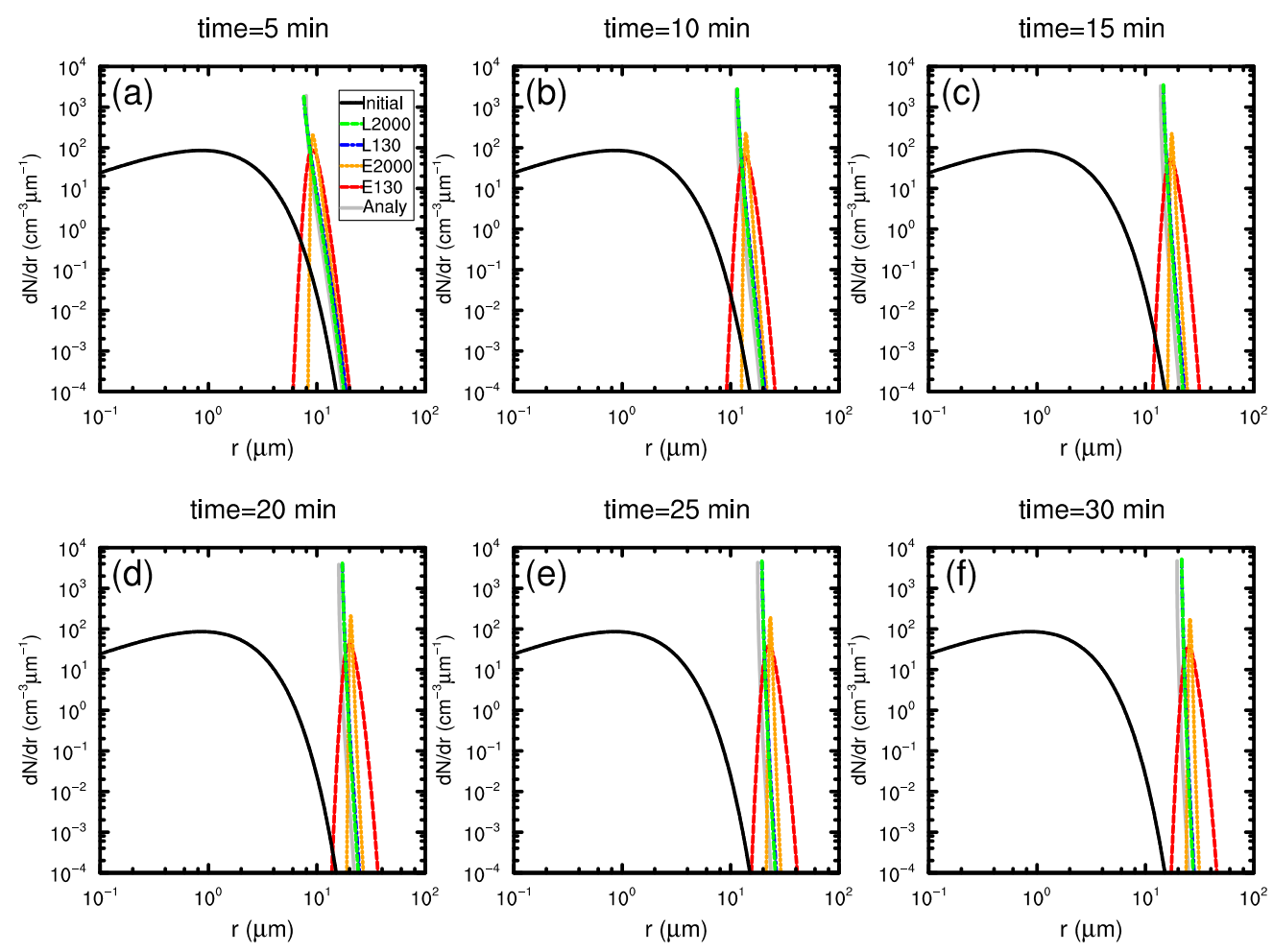

Figure 2. The time evolution of the droplet spectra in condensation growth from the analytical solution (Analy) and numerical solutions in the parcel cloud model: Eulerian advection scheme with 130 bins (E130), Eulerian advection scheme with 2000 bins (E2000), Lagrangian advection scheme with 130 bins (L130), Lagrangian advection scheme with 2000 bins (L2000), and the initial spectra (Initial). (a) Droplet spectra at $5 \mathrm{~min}$. (b) Droplet spectra at $10 \mathrm{~min}$. (c) Droplet spectra at $15 \mathrm{~min}$. (d) Droplet spectra at $20 \mathrm{~min}$. (e) Droplet spectra at $25 \mathrm{~min}$. (f) Droplet spectra at $30 \mathrm{~min}$.

It can be seen that the evolution of the shape and position of the spectra in Figure $2 b-f$ is not so obvious compared to those in Figure $2 \mathrm{a}$ as the growth rate for a single drop is inversely proportional to drop radius. As all droplets were assumed to be spherical, cloud drops with larger radii had larger surface area, so more mass was required to make a larger cloud drop grow. The position of the peak of the spectra from the LAS was quite close to those from the analytical solutions throughout the simulation. The peak reached $11.5 \mu \mathrm{m}$ at $10 \mathrm{~min}, 17.2 \mu \mathrm{m}$ at $20 \mathrm{~min}$, and $21.5 \mu \mathrm{m}$ at $30 \mathrm{~min}$ with LAS, slightly faster than the analytical solutions. Correspondingly, the peak reached $13.4 \mu \mathrm{m}$ at $10 \mathrm{~min}, 20.0 \mu \mathrm{m}$ at $20 \mathrm{~min}$, and $25.2 \mu \mathrm{m}$ at $30 \mathrm{~min}$ with EAS, significantly faster than the analytical solutions. The cloud drop spectra from EAS with 2000 bins were significantly narrower than those with 130 bins after $15 \mathrm{~min}$, supporting the previous point that spurious broadening can be alleviated but not eliminated with increasing number of bins. The spectra produced by LAS with 130 bins were almost identical to those with 2000 bins. In other words, increasing the bin numbers did not improve the performance of the scheme in the parcel cloud model. This characteristic can be exploited to reduce computational resources. 
In LAS, the bin width was explicitly advanced by solving Equation (6). Figure 3 shows the time evolution of the bin width from the LAS with 130 bins. Only the first 77 bins were assigned with cloud drops and advanced. As for the first 60 bins, the bin width decreased with time. For bins with large initial radii, the width almost remained constant due to extremely low growth rate. The width of bins with small initial radii reduced sharply in the first $5 \mathrm{~min}$ and reduced rather slowly after $10 \mathrm{~min}$. For instance, the width of the first bin was $1.20 \times 10^{-2} \mu \mathrm{m}$ at initial time, $1.66 \times 10^{-4} \mu \mathrm{m}$ at $5 \mathrm{~min}$, $1.09 \times 10^{-4} \mu \mathrm{m}$ at $10 \mathrm{~min}$, and $5.84 \times 10^{-5} \mu \mathrm{m}$ at $30 \mathrm{~min}$. The width of the 60 th bin was $11.00 \mu \mathrm{m}$ at initial time, $10.94 \mu \mathrm{m}$ at $5 \mathrm{~min}, 10.89 \mu \mathrm{m}$ at $10 \mathrm{~min}$, and $10.67 \mu \mathrm{m}$ at $30 \mathrm{~min}$. These characteristics can be taken into account in future cloud model developments.

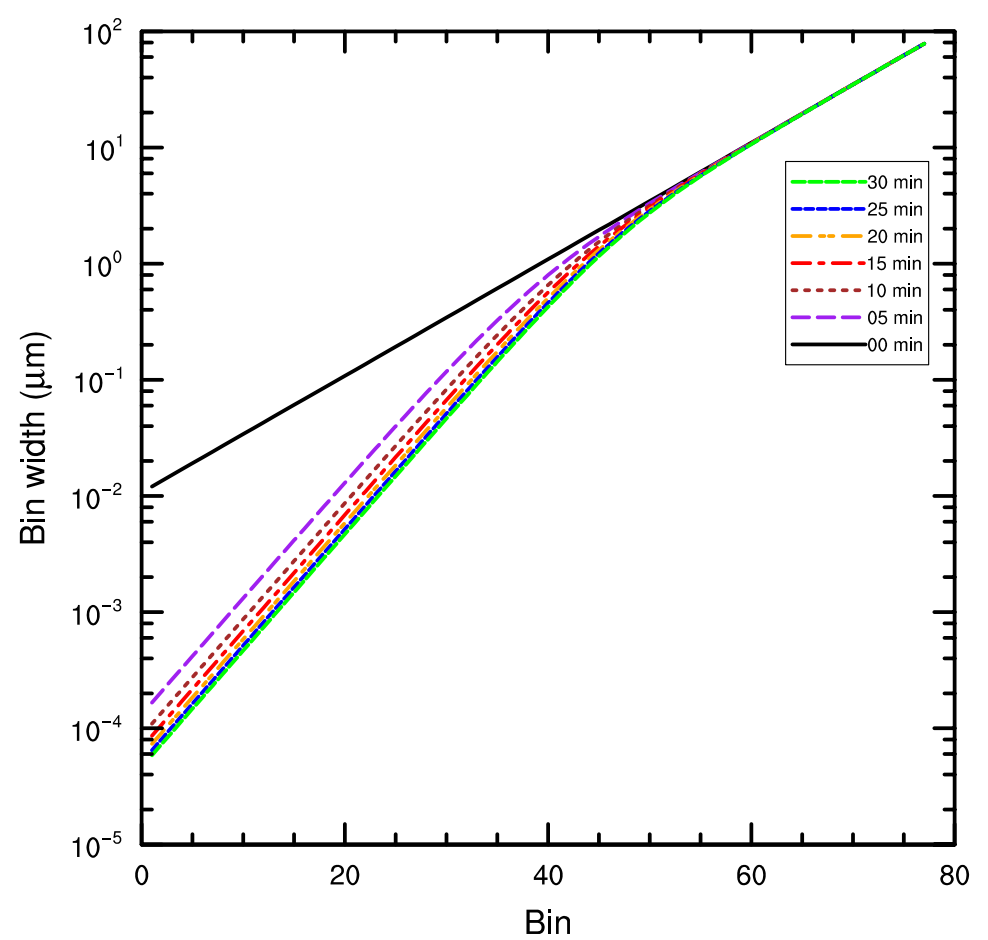

Figure 3. The time evolution of the bin width $\left(\Delta r_{i}\right)$ in the parcel cloud model with the Lagrangian advection scheme (130 bins).

Analysis of the results of the parcel model tests suggested that the new fully Lagrangian advection scheme could faithfully reproduce cloud drop spectrum evolution in the condensation growth process. Secondly, the LAS did not suffer the cloud drop spectrum spurious broadening issue as EAS did. The parcel cloud models with Eulerian advection schemes did not eliminate cloud drop spectrum spurious broadening even though up to 2000 bins were used. Finally, the new LAS was not sensitive to the bin resolution in numerical tests of cloud drop condensation growth. Modelers can therefore choose moderate bin number to achieve a balance between resolution and computational efficiency.

\subsection{One-and-Half Dimensional Cloud Model}

The bottom level of the model was set to $800 \mathrm{~m}$ above mean sea level (msl) to accommodate the sounding input data (which started from $821 \mathrm{~m}$ above $\mathrm{msl}$ ). The heights in this section refer to height above msl by default. Therefore, $800 \mathrm{~m}$ needed to be added to the model height before comparing observations. The simulated cloud base was at about $3.5 \mathrm{~km}$, and the maximum cloud top was at about $9.8 \mathrm{~km}$ with EAS and LAS. The observed cloud base was $3.9 \mathrm{~km}$, and maximum visual cloud top was about $10.5 \mathrm{~km}$ [34]. A similar discrepancy between the simulated and observed cloud base height was also reported in a CCOPE case study, in which it was attributed to the lack of representativeness of the input sounding data [34]. The simulated cloud depth was close to the observed cloud depth, although 
the ice-phase processes were blocked in the models. The ice-phase processes were excluded for two reasons. Firstly, liquid-phase processes rather than ice-phase processes play a dominant role in the ascent period of the sailplane as few ice particles are sampled [33]. Secondly, the focus of this numerical test was to evaluate LAS against EAS for solving cloud drop condensation (evaporation). Incorporating ice-phase processes into the simulations would probably complicate the picture. The simulated $0{ }^{\circ} \mathrm{C}$ level (about $3.8 \mathrm{~km}$ ) was also in agreement with the observations [33].

Figure 4 shows the vertical profile of the particle size distribution at 35 and $52.5 \mathrm{~min}$. The initial total number concentration of CCN particles decreased exponentially with height as prescribed by the model. The convective cell was at the early developing stage at $35 \mathrm{~min}$, with cloud base at about $3.5 \mathrm{~km}$ and cloud top at about $5.3 \mathrm{~km}$. The particle size distributions appeared in double-peaked structure at all levels within the cloud. The peak on the left side corresponded to CCN particles, while the second peak consisted of cloud drops. The second peak stretched slantwise vertically as the cloud top was a high value zone in supersaturation throughout the developing stage of the cloud. In general, the vertical profile of the particle size distribution with LAS was similar to those with EAS at $35 \mathrm{~min}$.
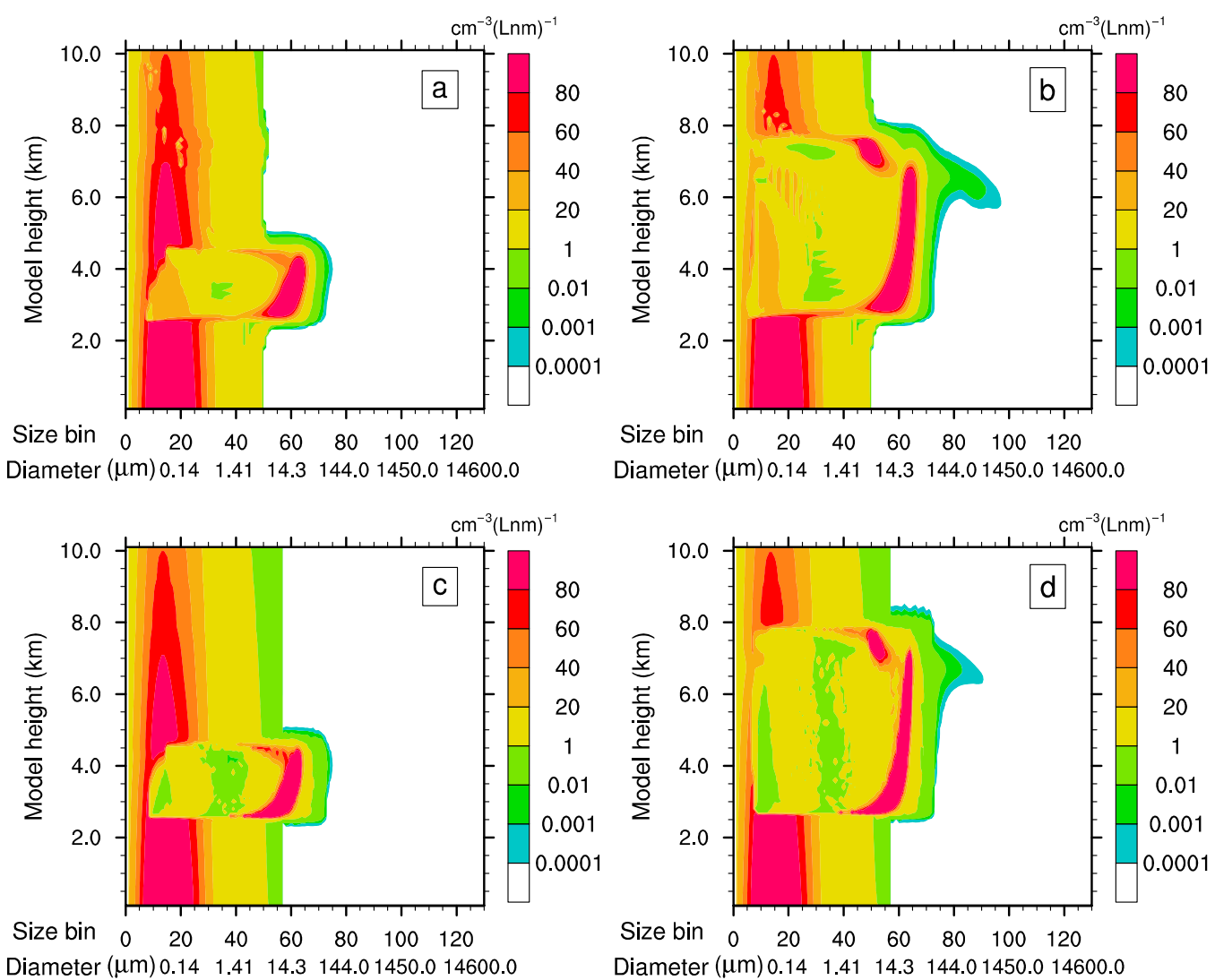

Figure 4. Number distributions of ammonium sulfate aerosol particles and water droplets as a function of height, the natural logarithm of the water mass of the bin, and the natural logarithm of the aerosol mass of the bin $f\left(\right.$ Lnm $_{\text {water }}, L_{n m}$ aerosol,$\left.Z\right)\left(\right.$ Number $\left.\mathrm{cm}^{-3}\left(\operatorname{Lnm}_{\text {water }}\right)^{-1}\right)$ at $35 \mathrm{~min}(\mathbf{a}, \mathbf{c})$ and $52.5 \mathrm{~min}(\mathbf{b}, \mathbf{d})$ with the Eulerian advection scheme $(\mathbf{a}, \mathbf{b})$ and with the Lagrangian advection scheme $(\mathbf{c}, \mathbf{d})$.

The convective cloud was at the late developing stage at $52.5 \mathrm{~min}$, with cloud base at about $3.5 \mathrm{~km}$ and cloud top at about $8.6 \mathrm{~km}$. Compared to the vertical profile at $35 \mathrm{~min}$, increasing number of $\mathrm{CCN}$ particles were activated due to accumulation of supersaturation as the cloud evolved. The double-peaked mode maintained due to the cloud droplet nucleation at the top interface of the cloud and cloud-free air [1], although the peak composed of CCN was significantly weakened. Distinctions between the results with LAS and those with EAS became evident, with the cloud droplet peak with LAS being narrower than that with EAS, especially at the upper part of the cumulus cloud. 
A common explanation for this is that cloud drops at the upper levels generally experience longer time of condensation after nucleation, and the effects of adopting different condensation schemes were therefore well exposed. Raindrops (diameter larger than $100 \mu \mathrm{m}$, approximately the 77th bin) existed at $52.5 \mathrm{~min}$ with both EAS and LAS. However, the number density of raindrops with diameter larger than $256 \mu \mathrm{m}$ (85th bin) was higher with EAS than with LAS. Vertical transportations of raindrops could also be observed.

Figure 5 shows the vertical distribution of cloud drop number concentrations and cloud drop mean diameter above the cloud base. The FSSP sample particles were 0.5 to $47.0 \mu \mathrm{m}$ in diameter. For comparison with observations, the particles from the 31st bin $(0.5 \mu \mathrm{m})$ to the 70th bin $(45.3 \mu \mathrm{m})$ were used to calculate the model results at $52.5 \mathrm{~min}$ (same as Figure 7a). The EAS gave a slightly better simulation of the particle number concentrations except at the lower levels, while the LAS overestimated the number concentrations at the middle and upper levels. The preset initial CCN concentrations in the model may deviate from the actual value and influence the simulated particle concentrations. The right-hand side panel of Figure 5 shows the particle mean diameter within the range of FSSP. The LAS gave an accurate reproduction of the mean diameter from the cloud base up to $3.2 \mathrm{~km}$, while the EAS overestimated it. Moreover, the deviations with EAS increased with height to more than $2 \mu \mathrm{m}$. The data were not sufficient to compare the LAS and EAS near the cloud top.
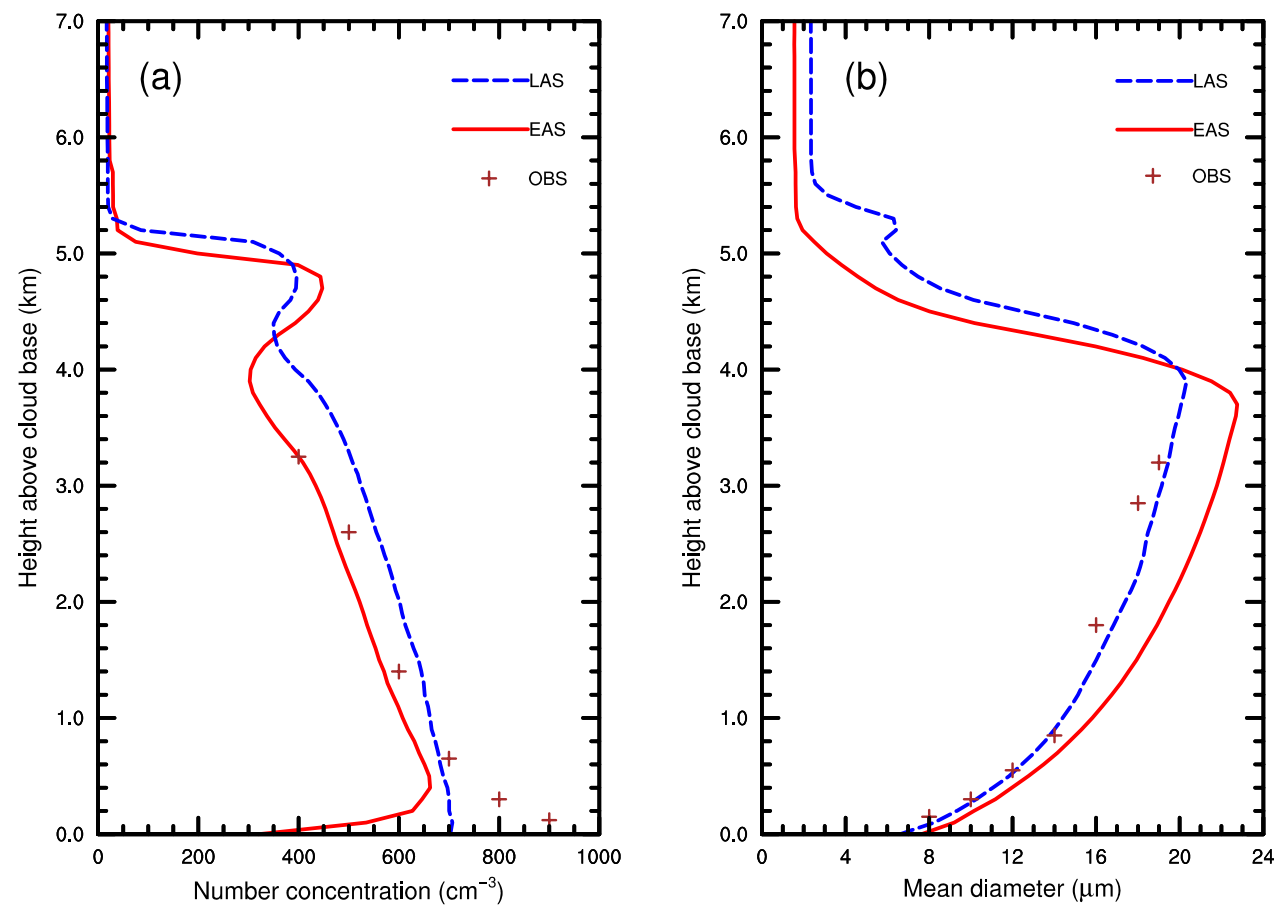

Figure 5. Comparisons between model results at $52.5 \mathrm{~min}$ with Lagrangian advection scheme (LAS), Eulerian advection scheme (EAS), and observations by the sailplane (OBS). (a) Particle number concentration. (b) Particle mean diameter.

Figure $6 a, c$ shows the evolution of the vertical profile of the liquid water content and the vertical wind. In general, the distribution pattern of the liquid water content with LAS was close to that with EAS. However, the maximum value with LAS was lower than that with EAS. The maximum value with EAS exceeded $2.0 \mathrm{~g} \cdot \mathrm{m}^{-3}$, while that with LAS was lower than $2.0 \mathrm{~g} \cdot \mathrm{m}^{-3}$. Lower concentrations of large cloud drops (diameter larger than $25 \mu \mathrm{m}$, on the right-hand side of the 65th bin) as well as raindrops with LAS might have in part accounted for this distinction. The time evolution of vertical wind profile with LAS was also close to that with EAS, with simulated maximum speed between 10 and $12 \mathrm{~m} \cdot \mathrm{s}^{-1}$. Figure $6 \mathrm{~b}$,d shows the evolution of the vertical profile of the supersaturation ratio and the temperature. Two zones of high supersaturation values could be observed, one at the cloud 
base and the other one at the cloud top. The peak value at the cloud base has been found in previous studies by parcel cloud models [35-37]. However, one theory claims that the expansion and cooling of ascending moist air parcel above the cloud top results in the cloudy air becoming supersaturated with condensation rather than evaporation at the interface of cloudy air and clear air [1]. It should also be noted that the supersaturation ratio with LAS was noticeably lower than that with EAS at the cloud base. Note that the supersaturation is determined by both the vertical velocity and the condensation rate. Since the little difference of velocities and the big difference of cloud droplet concentrations at the cloud base lead to much more consumption for supersaturation water vapor with high droplet concentrations in the simulation with LAS, it is reasonable that the supersaturation ratio with LAS was lower than that with EAS at the cloud base.
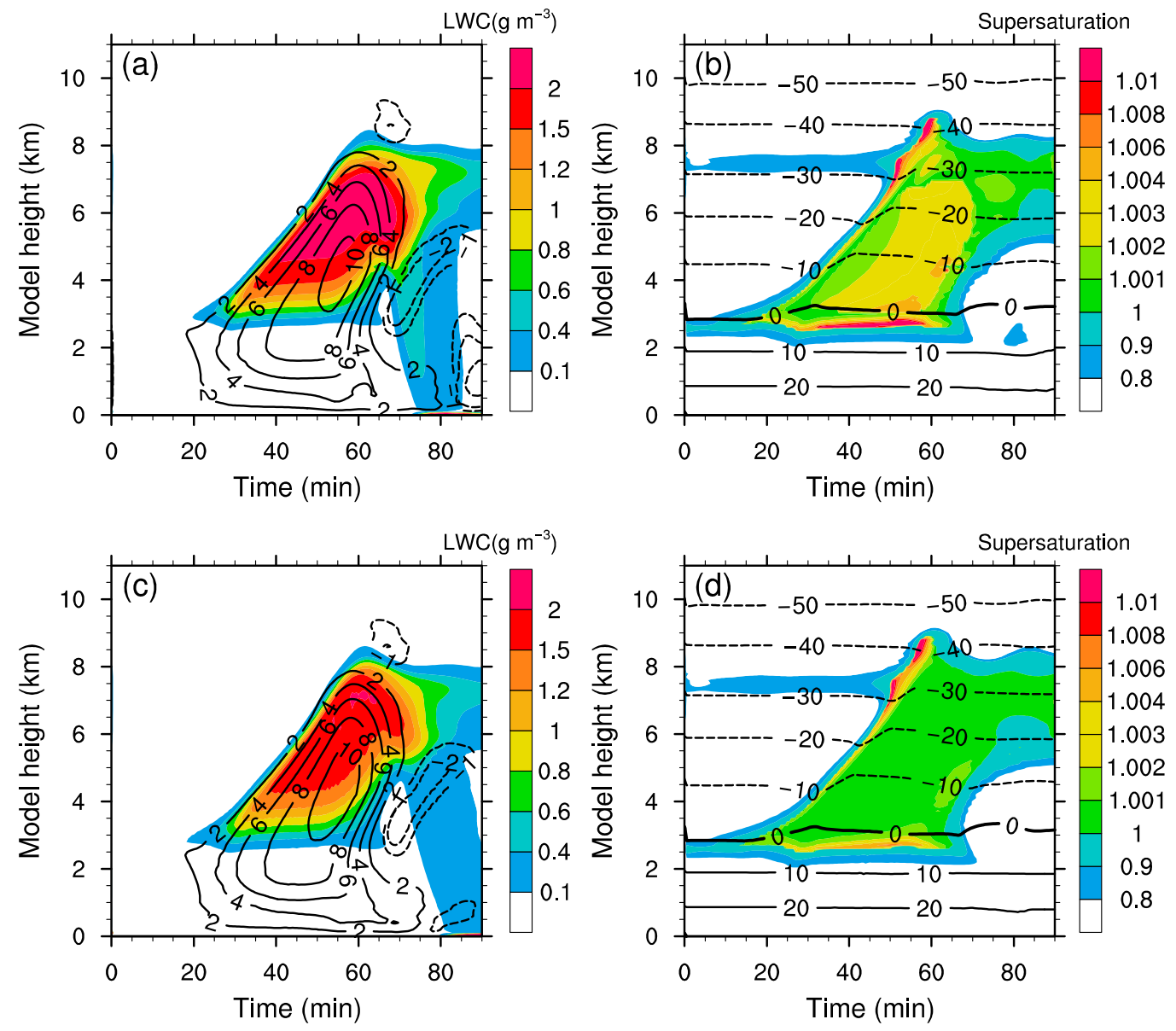

Figure 6. Spatial and temporal evolution of the thermodynamic variables with the Eulerian advection scheme $(\mathbf{a}, \mathbf{b})$ and the Lagrangian advection scheme $(\mathbf{c}, \mathbf{d}) .(\mathbf{a}, \mathbf{c})$ Liquid water content (shaded area) and vertical wind (solid and dashed lines) (unit: $\mathrm{g} \cdot \mathrm{m}^{-3}$ and $\mathrm{m} \cdot \mathrm{s}^{-1}$ ) as a function of time and height. (b,d) Supersaturation ratio (shaded area) and temperature (solid and dashed lines) (unit: none and degree Celsius) as a function of time and height.

Figure 7 shows the vertical distribution of liquid water content sampled by FSSP and vertical wind above the cloud base. The EAS gave a slightly better representation of the liquid water content around the cloud base and the cloud top, while the LAS performed much better in general. This is consistent with the simulation results of particle mean diameter in Figure 5. A straightforward explanation of this is that the overestimation of particle mean diameter with EAS resulted in the overestimation of liquid water content. The right-hand side panel of Figure 7 shows the simulated and observational vertical wind distributions. Both the EAS and LAS overestimated the wind velocity at the lower and middle 
levels. However, the representativeness of the observed vertical wind by sailplane can be weakened due to the strong impulse nature.
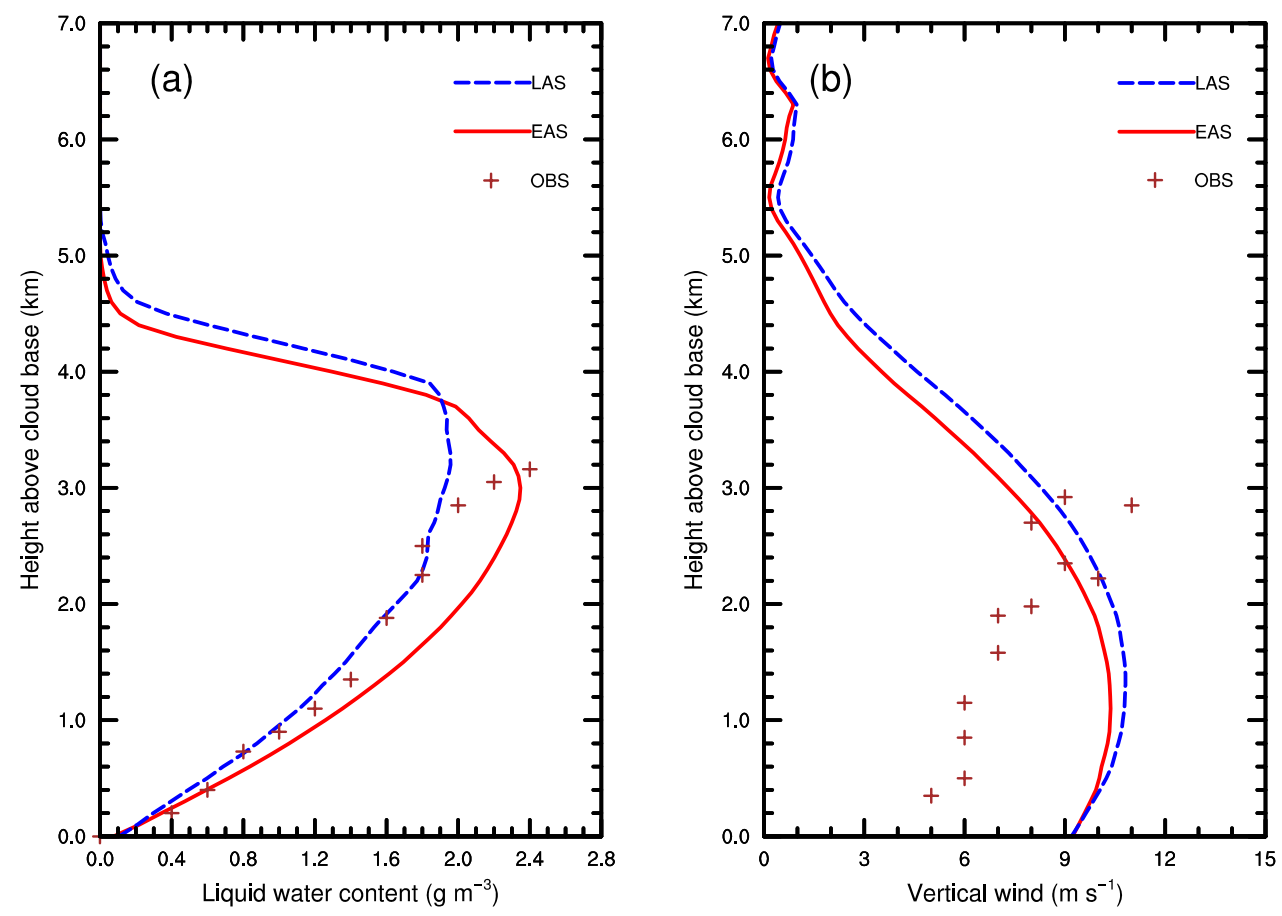

Figure 7. Comparisons between model results at $52.5 \mathrm{~min}$ with LAS, EAS, and OBS. (a) Liquid water content. (b) Vertical wind.

\section{Conclusions}

The main purpose of this work was to introduce a new fully Lagrangian advection scheme for solving the time evolution of cloud drop size distribution through water vapor diffusion growth. Movable bins were used to discretize and advance CDSD in the LAS. The radius and width of bins were obtained by numerically integrating the cloud drop diffusion growth equation and the newly derived equation. CDSD was then diagnosed at each timestep by combining information about the radius of bins, width of bins, and the cloud drop number concentration on each bin. Theoretically, the position of the mobile units (bins) as well as the deformation of the units should be advanced by a Lagrangian advection scheme simultaneously. In the scenario of simulations of cloud drop diffusion growth, the evolution of the width of bins (mobile units) can therefore be argued as an integral part of the Lagrangian advection scheme.

The Lagrangian advection scheme was tested in a parcel cloud model. The time evolution of the cloud drop spectrum simulated with the new scheme was in good accordance with the reference analytical solutions. We can conclude that, firstly, the new scheme can accurately reproduce the cloud drop diffusion growth process in a parcel cloud model. Secondly, the performance of the new scheme is not sensible to the number of bins used, which can be utilized by modelers to save computational resources. Thirdly, the long-standing cloud drop spectrum spurious broadening issue in Eulerian advection schemes, which can be alleviated but not eliminated at the cost of increasing bin numbers, does not affect the new Lagrangian advection scheme.

Bridged by an interpolation algorithm, the new scheme was further coupled into a one-and-half dimensional cloud-aerosol bin model to test its performance. Numerical convection tests for an isolated deep cumulus cloud in the CCOPE campaign showed that the LAS produced steeper cloud droplet spectra than those with the EAS, suggesting that the new scheme contributes to reducing artificial CDSD broadening. Moreover, the LAS significantly improved the simulation results of particle mean 
diameter and liquid water content within the sampling range of FSSP. It can be concluded that the overall performance of the 1.5D model improved with LAS in this case study.

In future work, efforts should be made to couple the Lagrangian advection scheme into a three-dimensional cloud model with complete warm rain processes covered. Case studies and numerical investigations will be conducted focusing on the formation of warm rain embryos and the initiation of collision-coalescence process in warm clouds.

Author Contributions: Conceptualization: J.S. and L.D.; formal analysis: L.W. and W.H.; investigation: L.W. and W.H.; methodology: W.H., J.S. and L.D.; project administration: L.W., H.L. and J.S.; software: L.W., L.D. and W.H.; funding acquisition: L.W., and H.L.; writing (original draft): L.W. and W.H.; writing (review and editing): L.W., J.S. and W.H. All authors have read and agreed to the published version of the manuscript.

Funding: This research was funded by the National Natural Science Foundation of China, grant number 41705119 and 41530427.

Acknowledgments: We would like to thank everyone who has supported and helped this project.

Conflicts of Interest: The authors declare no conflict of interest.

\section{References}

1. Sun, J.; Leighton, H.; Yau, M.K.; Ariya, P. Numerical evidence for cloud droplet nucleation at the cloud-environment interface. Atmos. Chem. Phys. Discuss. 2012, 12, 12155-12164. [CrossRef]

2. Khain, A.; Beheng, K.D.; Heymsfield, A.; Korolev, A.; Krichak, S.O.; Levin, Z.; Pinsky, M.; Phillips, V.; Prabhakaran, T.; Teller, A.; et al. Representation of microphysical processes in cloud-resolving models: Spectral (bin) microphysics versus bulk parameterization. Rev. Geophys. 2015, 53, 247-322. [CrossRef]

3. Khain, A.P. Notes on state-of-the-art investigations of aerosol effects on precipitation: A critical review. Environ. Res. Lett. 2009, 4, 15004. [CrossRef]

4. Tao, W.-K.; Chen, J.; Li, Z.; Wang, C.; Zhang, C. Impact of aerosols on convective clouds and precipitation. Rev. Geophys. 2012, 50, 50. [CrossRef]

5. Kessler, E. On the continuity and distribution of water substance in atmospheric circulations. Atmos. Res. 1995, 38, 109-145. [CrossRef]

6. Clark, T.L. Numerical Modeling of the Dynamics and Microphysics of Warm Cumulus Convection. J. Atmos. Sci. 1973, 30, 857-878. [CrossRef]

7. Khain, A.; Pokrovsky, A. Simulation of Effects of Atmospheric Aerosols on Deep Turbulent Convective Clouds Using a Spectral Microphysics Mixed-Phase Cumulus Cloud Model. Part II: Sensitivity Study. J. Atmos. Sci. 2004, 61, 2983-3001. [CrossRef]

8. Thompson, G.; Rasmussen, R.M.; Manning, K. Explicit Forecasts of Winter Precipitation Using an Improved Bulk Microphysics Scheme. Part I: Description and Sensitivity Analysis. Mon. Weather Rev. 2004, 132, 519-542. [CrossRef]

9. Saleeby, S.M.; Cotton, W.R. A Large-Droplet Mode and Prognostic Number Concentration of Cloud Droplets in the Colorado State University Regional Atmospheric Modeling System (RAMS). Part I: Module Descriptions and Supercell Test Simulations. J. Appl. Meteorol. 2004, 43, 182-195. [CrossRef]

10. Milbrandt, J.A.; Yau, M.K. A Multimoment Bulk Microphysics Parameterization. Part III: Control Simulation of a Hailstorm. J. Atmos. Sci. 2006, 63, 3114-3136. [CrossRef]

11. Loftus, A.; Cotton, W. Examination of CCN impacts on hail in a simulated supercell storm with triple-moment hail bulk microphysics. Atmos. Res. 2014, 147, 183-204. [CrossRef]

12. Pruppacher, P.S.; Klett, J.D. Microphysics of Clouds and Precipitation; Kluwer Academic: Dordrecht, The Nederlands, 1997; p. 954.

13. Segal, Y.; Khain, A.; Pinsky, M.; Rosenfeld, D. Effects of hygroscopic seeding on raindrop formation as seen from simulations using a 2000-bin spectral cloud parcel model. Atmos. Res. 2004, 71, 3-34. [CrossRef]

14. Stevens, B.; Walko, R.L.; Cotton, W.R.; Feingold, G. The Spurious Production of Cloud-Edge Supersaturations by Eulerian Models. Mon. Weather Rev. 1996, 124, 1034-1041. [CrossRef]

15. Naumann, A.K.; SeifertiD, A. A Lagrangian drop model to study warm rain microphysical processes in shallow cumulus. J. Adv. Model. Earth Syst. 2015, 7, 1136-1154. [CrossRef] 
16. Andrejczuk, M.; Reisner, J.M.; Henson, B.; Dubey, M.K.; Jeffery, C.A. The potential impacts of pollution on a nondrizzling stratus deck: Does aerosol number matter more than type? J. Geophys. Res. 2008, 113, D19204. [CrossRef]

17. Shima, S.; Kusano, K.; Kawano, A.; Sugiyama, T.; Kawahara, S. The super-droplet method for the numerical simulation of clouds and precipitation: A particle-based and probabilistic microphysics model coupled with a non-hydrostatic model. Q. J. R. Meteorol. Soc. 2009, 135, 1307-1320. [CrossRef]

18. Riechelmann, T.; Noh, Y.; Raasch, S. A new method for large-eddy simulations of clouds with Lagrangian droplets including the effects of turbulent collision. New J. Phys. 2012, 14, 65008. [CrossRef]

19. Li, X.-Y.; Brandenburg, A.; Haugen, N.E.; Svensson, G. Eulerian and L agrangian approaches to multidimensional condensation and collection. J. Adv. Model. Earth Syst. 2017, 9, 1116-1137. [CrossRef]

20. Onishi, R.; Matsuda, K.; Takahashi, K. Lagrangian Tracking Simulation of Droplet Growth in Turbulence-Turbulence Enhancement of Autoconversion Rate. J. Atmos. Sci. 2015, 72, 2591-2607. [CrossRef]

21. Saito, I.; Gotoh, T. Turbulence and cloud droplets in cumulus clouds. New J. Phys. 2018, 20, 23001. [CrossRef]

22. Chen, S.; Yau, M.-K.; Bartello, P.; Xue, L. Bridging the condensation-Collision size gap: A direct numerical simulation of continuous droplet growth in turbulent clouds. Atmos. Chem. Phys. Discuss. 2018, 18, 7251-7262. [CrossRef]

23. Kunishima, Y.; Onishi, R. Direct Lagrangian tracking simulation of droplet growth in vertically developing cloud. Atmos. Chem. Phys. Discuss. 2018, 18, 16619-16630. [CrossRef]

24. Pinsky, M.B.; Khain, A. Effects of in-cloud nucleation and turbulence on droplet spectrum formation in cumulus clouds. Q. J. R. Meteorol. Soc. 2002, 128, 501-533. [CrossRef]

25. Dong, L.; Wang, B.; Liu, L. A Lagrangian advection scheme with shape matrix (LASM) for solving advection problems. Geosci. Model Dev. 2014, 7, 2951-2968. [CrossRef]

26. Rogers, R.R.; Mason, B.J.; Sartor, J.D. A Short Course in Cloud Physics and Clouds, Rain, and Rainmaking, 2nd Edition. Phys. Today 1976, 29, 52. [CrossRef]

27. Khain, A.; Ovtchinnikov, M.; Pinsky, M.; Pokrovsky, A.; Krugliak, H. Notes on the state-of-the-art numerical modeling of cloud microphysics. Atmos. Res. 2000, 55, 159-224. [CrossRef]

28. Schär, C.; Smolarkiewicz, P.K. A Synchronous and Iterative Flux-Correction Formalism for Coupled Transport Equations. J. Comput. Phys. 1996, 128, 101-120. [CrossRef]

29. Smolarkiewicz, P.K.; Grabowski, W.W. The multidimentional positive definite advection transport algorithm: Nonoscillatory option. J. Comput. Phys. 1990, 86, 355-375. [CrossRef]

30. Sun, J.; Ariya, P.A.; Leighton, H.G.; Yau, M.K. Modeling Study of Ice Formation in Warm-Based Precipitating Shallow Cumulus Clouds. J. Atmos. Sci. 2012, 69, 3315-3335. [CrossRef]

31. Leroy, D.; Monier, M.; Wobrock, W.; Flossmann, A. A numerical study of the effects of the aerosol particle spectrum on the development of the ice phase and precipitation formation. Atmos. Res. 2006, 80, 15-45. [CrossRef]

32. Sun, J. Ice Initiation and Ice Multiplication Processes in a Warm-Based Precipitating Cumulus Cloud Model. Ph.D. Thesis, McGill University, Montreal, QC, Canada, 2008.

33. Dye, J.E.; Jones, J.J.; Winn, W.P.; Cerni, T.A.; Gardiner, B.; Lamb, D.; Pitter, R.L.; Hallett, J.; Saunders, C.P.R. Early electrification and precipitation development in a small, isolated Montana cumulonimbus. J. Geophys. Res. 1986, 91, 1231. [CrossRef]

34. Masataka, M. Numerical Modeling of Dynamical and Microphysical Evolution of an Isolated Convective Cloud-The 19 July 1981 CCOPE Cloud. J. Meteorol. Soc. Jpn. 1990, 68, 107-128.

35. Beard, K.V.; Ochs, H.T. Warm-rain initiation: An overview of microphysical mechanisms. J. Appl. Meteor. 1993, 32, 608-625. [CrossRef]

36. Segal, Y.; Pinsky, M.; Khain, A.; Erlick, C. Thermodynamic factors influencing bimodal spectrum formation in cumulus clouds. Atmos. Res. 2003, 66, 43-64. [CrossRef]

37. Segal, Y.; Pinsky, M.; Khain, A. The role of competition effect in the raindrop formation. Atmos. Res. 2007, 83, 106-118. [CrossRef]

(C) 2020 by the authors. Licensee MDPI, Basel, Switzerland. This article is an open access article distributed under the terms and conditions of the Creative Commons Attribution (CC BY) license (http://creativecommons.org/licenses/by/4.0/). 